\title{
Global Assessment of Cardiac Function Using Image Statistics in MRI
}

\author{
Mariam Afshin ${ }^{1,3}$, Ismail Ben Ayed ${ }^{2}$, Ali Islam ${ }^{1}$, Aashish Goela ${ }^{1}$, \\ Terry M. Peters ${ }^{1,3}$, and Shuo $\mathrm{Li}^{2}$ \\ 1 University of Western Ontario, London, Canada \\ 2 GE Healthcare, London, Canada \\ 3 Robarts Research Institute, London, Canada
}

\begin{abstract}
The cardiac ejection fraction (EF) depends on the volume variation of the left ventricle (LV) cavity during a cardiac cycle, and is an essential measure in the diagnosis of cardiovascular diseases. It is often estimated via manual segmentation of several images in a cardiac sequence, which is prohibitively time consuming, or via automatic segmentation, which is a challenging and computationally expensive task that may result in high estimation errors. In this study, we propose to estimate the EF in real-time directly from image statistics using machine learning technique. From a simple user input in only one image, we build for all the images in a subject dataset (200 images) a statistic based on the Bhattacharyya coefficient of similarity between image distributions. We demonstrate that these statistics are non-linearly related to the LV cavity areas and, therefore, can be used to estimate the EF via an Artificial Neural Network (ANN) directly. A comprehensive evaluation over 20 subjects demonstrated that the estimated EFs correlate very well with those obtained from independent manual segmentations.
\end{abstract}

\section{Introduction}

One of the most important observations in diagnosing cardiovascular diseases, the cardiac ejection fraction (EF), may decrease in the case of a heart attack or other problems related to the heart valves or muscles. Furthermore, EF is an important indicator of long-term prognosis for patients with coronary artery disease. Because the left ventricle $(\mathrm{LV})$ is the main pumping chamber of the heart, EF is usually measured using information from the LV [7]. In routine clinical use, it is often estimated from several images in a cardiac sequence using manual segmentation of the LV cavity, which is prohibitively time consuming. While automatic LV segmentation can be used to compute the EF, automatic LV segmentation is still acknowledged as a challenging, computationally expensive task, which has attracted impressive research attention in recent years. Existing LV segmentation algorithms are based on traditional techniques, such as thresholding, region-growing, edge detection and clustering [8, 9, 11], and energy minimization techniques such as graph cuts [4, 13], active contours/level sets [2, 6], as well as active appearance and shape models [1]. In general, segmentation algorithms require a careful user initialization, intensive training, and a heavy computational load. Furthermore, the ensuing segmentation results depend significantly on the choice of a set of ad hoc parameters and training data, which may yield high errors in the computation of the

N. Ayache et al. (Eds.): MICCAI 2012, Part II, LNCS 7511, pp. 535-543, 2012.

(c) Springer-Verlag Berlin Heidelberg 2012 
EF. These difficulties, inherent to segmentation algorithms, impede the automatic estimation of the $\mathrm{EF}$ in routine clinical use. A recent comprehensive review of cardiac image segmentation and its challenges can be found in [12]. While existing techniques are labor intensive, we believe that there are other characteristics of the images that can be computed with less effort, but that nevertheless correlate strongly with the EF. One such technique that we describe below is based on machine learning, which removes the need for image segmentation. From a simple user input in one single image, we build for all the images in a subject dataset (200 images) a statistic based on the Bhattacharyya coefficient [3] of similarity between image distributions. We demonstrate that these statistics are non-linearly related to the LV cavity areas (cf. Fig. 3) and therefore can be used to estimate the EF directly via an Artificial Neural Network (ANN). The proposed method consists of four main steps: (1) Image acquisition, (2) Building Image Statistics, (3) Applying Artificial Neural Network, and (4) Estimating Ejection Fraction. A comprehensive quantitative evaluation over 20 subjects demonstrates that the estimated EFs correlated very well with those obtained from manual segmentations.

\section{Estimating Left Ventricle Volumes from Image Statistics}

\subsection{Building Image Statistics}

Let $\mathscr{I}$ be a cardiac MRI sequence containing $J$ frames 1 , each comprising $I$ slices, $\mathscr{I}_{i, j}: \Omega \subset \mathfrak{R}^{2} \rightarrow \mathfrak{R}^{+}$with $(i, j) \in[1 \ldots I] \times[1 \ldots J]$. To introduce our methodology for building an image statistic related to the LV cavity area for each image $\mathscr{I}_{i, j},(i, j) \in$ $[1 \ldots I] \times[1 \ldots J]$, let us consider the following definitions. (1) Let $\mathbf{I}$ be a reference image which we use for a simple user input (refer to the middle image in Fig.11b). For instance, in the experiments of this study, we used image $\mathscr{I}_{7,1}$ in each subject dataset. (2) Let $\Gamma_{\text {in }}, \Gamma_{\text {out }}:[0,1] \rightarrow \Omega$ denote two simple planar closed curves (e.g. squares) superimposed by the user on the reference image $\sqrt{3} \mathbf{I}$ (refer to the middle image in Fig. 1 b), one placed within the cavity (the blue curve in Fig. 1 b) and the other enclosing the cavity (the red curve). Let us now superimpose systematically (without additional user effort) $\Gamma_{\text {out }}$ onto each of the images in the subject dataset, as shown in Fig. 1. Then, we compute for each image a statistic based on the Bhattacharyya coefficient of similarity between image distributions (refer to Fig. 2), and demonstrate that the obtained statistics are related to the areas of the $L V$. Let $\mathbf{R}_{\Gamma} \subset \Omega$ be the region enclosed within $\Gamma, \Gamma \in\left\{\Gamma_{\text {in }}, \Gamma_{\text {out }}\right\}$, and $P_{\mathbf{R}_{\Gamma}, I}$ the kernel density estimate of the distribution of an image $I \in \mathscr{I}_{i, j},(i, j) \in$ $[1 \ldots I] \times[1 \ldots J]$, within region $\mathbf{R}_{\Gamma}$ :

$$
P_{\mathbf{R}_{\Gamma}, I}(z)=\frac{\int_{\mathbf{R}_{\Gamma}} K(z-I) d x}{a_{\mathbf{R}_{\Gamma}}}, \quad a_{\mathbf{R}_{\Gamma}}=\int_{\mathbf{R}_{\Gamma}} d x, \quad K(y)=\frac{1}{\sqrt{2 \pi \sigma^{2}}} \exp ^{-\frac{y^{2}}{2 \sigma^{2}}}
$$

where $a_{\mathbf{R}_{\Gamma}}$ is the area inside region $\mathbf{R}_{\Gamma}$ and $K$ is the Gaussian kernel [10]. We consider the distribution of the image within the region enclosed by the blue curve in the

\footnotetext{
1 The number of frames $J$ is typically equal to 20 or 25 .

2 The number of slices $I$ is typically equal to 10 .

${ }^{3}$ The reference image is a mid-cavity slices at the end-diastolic time.
} 


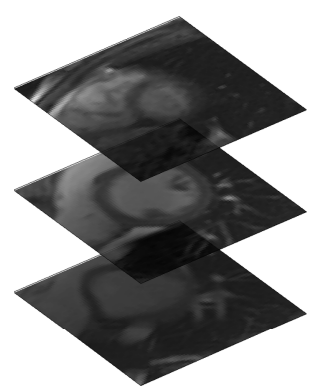

(a)

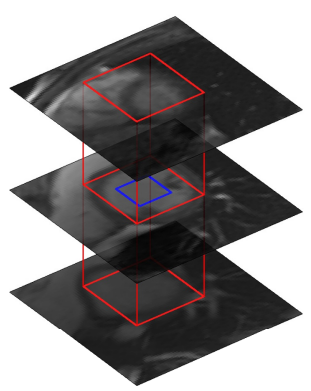

(b)

Fig. 1. (a) frame 1 (slices 1,7, and 10); (b) $\Gamma_{\text {in }}$ (the blue curve within the cavity) and $\Gamma_{\text {out }}$ (the red curve enclosing the cavity) are given by the user in the reference image (the middle image). $\Gamma_{i n}$ is used solely in the reference image to compute $P_{\mathbf{R}_{\Gamma_{i n}}, \mathbf{I}}$, whereas $\Gamma_{\text {out }}$ is superimposed systematically (without additional user effort) to all the other images to compute $P_{\mathbf{R}_{\Gamma_{\text {out }}}, \mathscr{I}_{i, j}}$.

reference image $\left(P_{\mathbf{R}_{\Gamma_{i n}}}, \mathbf{I}\right)$ as an approximation of the distribution within the cavity, and the distribution of the region enclosed by the red curve in each image $\mathscr{I}_{i, j}\left(P_{\mathbf{R}_{\Gamma_{\text {out }}}, \mathscr{I}_{i, j}}\right)$ as an approximation of the distribution of the entire left ventricle. Now consider the following measure of similarity between these two distributions in each image $\mathscr{I}_{i, j}$, $(i, j) \in[1 \ldots I] \times[1 \ldots J]:$

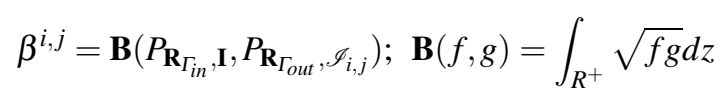

where the Bhattacharyya coefficient $\mathbf{B}(f, g)$ measures the amount of overlap (similarity) between two distributions $f$ and $g$. The range of the Bhattacharyya coefficient is $[0 ; 1]$, with 0 indicating no overlap between the distributions and 1 being a perfect match. The fixed $[0 ; 1]$ range of the Bhattacharyya coefficient affords a conveniently practical appraisal of the similarity. More importantly, we expect the measure $\beta^{i, j}$ to be related to the cavity area in the corresponding image $\mathscr{I}_{i, j}$. This is demonstrated experimentally by the typical example in Fig. 2, and the corresponding variations of the cavity areas and the Bhattacharyya statistics in Fig. 3 . Note the strong similarity between the variations of the cavity areas and those of the Bhattacharyya statistics (Fig. 3). Such similarity is reasonable since the more the distributions of the cavity and the LV overlap, the higher the cavity area.

\subsection{Artificial Neural Network (ANN) Estimation of LV Cavity Areas}

We constructed an Artificial Neural Network (ANN) to determine the nonlinear relation between the Bhattacharyya coefficients and the corresponding LV cavity areas (refer to Fig. 3 for an illustration of such non-linear relation). Following a back propagation ANN, a powerful machine learning technique [5], our feed-forward network consists of five layers, three hidden, one input, and one output (refer to Fig 4 (a) for an illustration). Let $P_{1,200}$ be the input of the network, a single row matrix containing the Bhattacharyya statistics, and let $T_{1,200}$ an output matrix containing the LV cavity areas: 


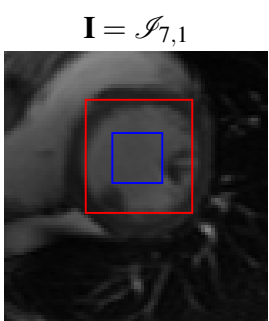

(a)

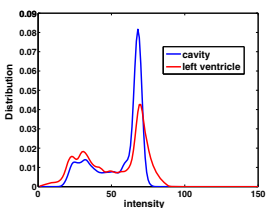

(d)

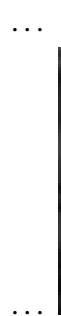

$\cdots$$$
\text { . }
$$

$\beta^{7,1}=0.9188$
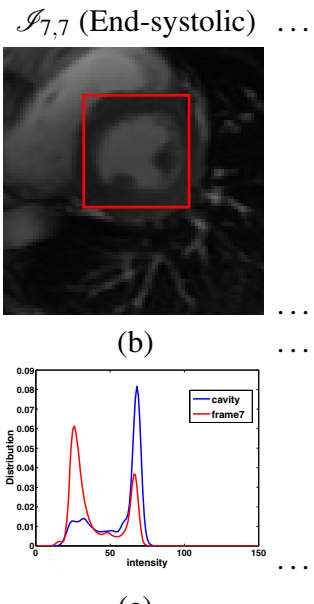

(b)

(e)

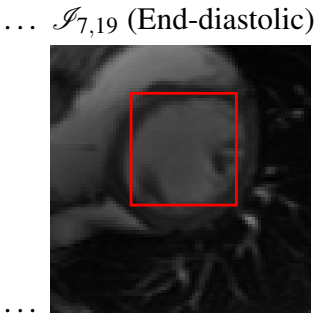

(c)

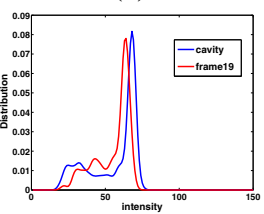

(f)

Fig. 2. Computing image statistics for the frames of slice 7 (middle slice): (a) reference image (red curve: $\Gamma_{\text {out }}$, blue curve: $\Gamma_{\text {in }}$ ); (b): frame 7 (end-systolic) and (c) frame 19 (end-diastolic); (d), (e), and (f) the corresponding distributions and Bhattacharyya measures $\left(\beta^{i j}\right)$. We observe that the variations of $\beta^{i j}$ are similar to the variations of the LV cavity areas. For instance at the end of systole (the middle column), the smallest cavity area coincides with the lowest Bhattacharyya measure.

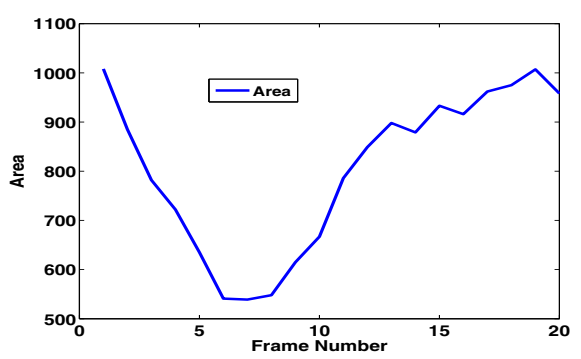

(a)

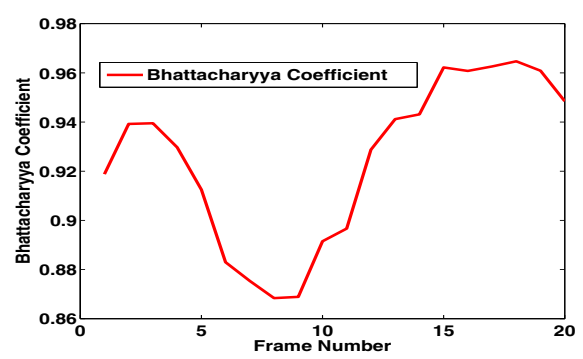

(b)

Fig. 3. (a) A typical example which shows the variations of LV cavity areas obtained from manual segmentations; (b) A typical example which shows the variations of the Bhattacharyya image statistics

$$
\begin{aligned}
& P_{1,200}=\left[P^{1}, \ldots, P^{j}, \ldots, P^{10}\right] \quad \text { with } \quad P^{j}=\left[\beta^{1, j} \ldots \beta^{i, j} \ldots \beta^{20, j}\right] \\
& T_{1,200}=\left[T^{1}, \ldots, T^{j}, \ldots, T^{10}\right] \quad \text { with } \quad T^{j}=\left[a^{1, j} \ldots a^{i, j} \ldots a^{20, j}\right]
\end{aligned}
$$

To reduce the dimensionality of the inputs and outputs, we used principal component analysis (PCA) to transform 200 possibly correlated variables into a smaller set of uncorrelated variables (the first five components in our case):

$$
I N_{1,5}=\operatorname{PCACOV}\left(P_{1,200}\right) \quad O U T_{1,5}=\operatorname{PCACOV}\left(T_{1,200}\right),
$$


where PCACOV is the PCA transform function, $I N_{1,5}$ and $O U T_{1,5}$ denote the transferred inputs and outputs respectively.

The next step is to train the network using the transferred inputs $\left(I N_{1,5}\right)$ and outputs $\left(O U T_{1,5}\right)$. The network we built estimates the following non-linear mapping: $O U T_{1,5}=$ $\mathbf{F}\left(I N_{1,5}\right)$ where $\mathbf{F}$ is a nonlinear transfer function consisting of two hyperbolic tangent functions and a linear function, a common choice in the neural network literature [5]. As illustrated in Fig 4 (a), the resulting network consists of five layers, one input and one output containing 5 neurons each, both based on the linear function $(f(x)=x)$, as well as three hidden layers containing 50, 25 and 50 neurons and based on the hyperbolic tangent, hyperbolic tangent and linear functions respectively. Let $I N P U T_{5,19}$ and

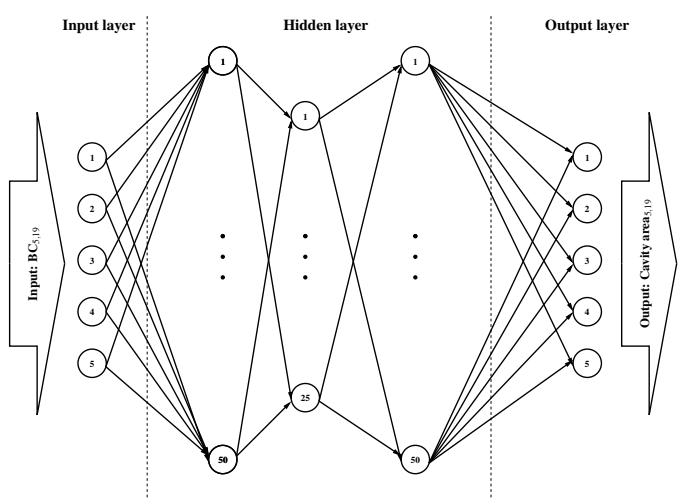

(a)

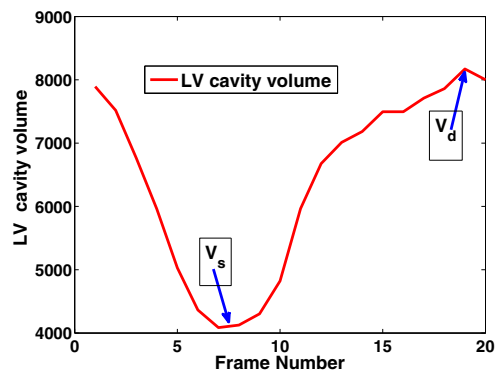

(b)

Fig. 4. (a) The ANN consists of one input layer, three hidden layers, and one output layer, (b) Variation of the volume of the LV cavity in each heart beat

OUTPUT $T_{5,19}$ denote the training input and output of the neural network respectively:

$$
\operatorname{INPUT} T_{5,19}=\left[\left(I N^{1}\right)^{-1}, \ldots,\left(I N^{a}\right)^{-1}, \ldots,\left(I N^{19}\right)^{-1}\right] \quad \text { st. } \quad I N^{a}{ }_{1,5}=\operatorname{PCACOV}\left(P_{1,200}\right)
$$

$$
\text { OUTPUT } T_{5,19}=\left[\left(O U T^{1}\right)^{-1}, \ldots,\left(O U T^{a}\right)^{-1}, \ldots,\left(O U T^{19}\right)^{-1}\right]
$$

$$
\text { st. } \quad O U T_{1,5}^{a}=\operatorname{PCACOV}\left(T_{1,200}\right)
$$

To validate this procedure, we employ a leave-one subject-out approach, where the test dataset was excluded from the training data. For the current testing subject dataset, the LV cavity areas were estimated using the transferred subject Bhattacharyya statistics and the learned non-linear mapping $\mathbf{F}$ as depicted in Fig.5(b).

$$
\text { OUT }_{\text {test } 1,5}=\mathbf{F}\left(\text { IN }_{\text {test } 1,5}\right) \quad T_{\text {test } 1,200}=\mathbf{P C A C O V}^{-1}\left(\text { OUT }_{\text {test } 1,5}\right)
$$




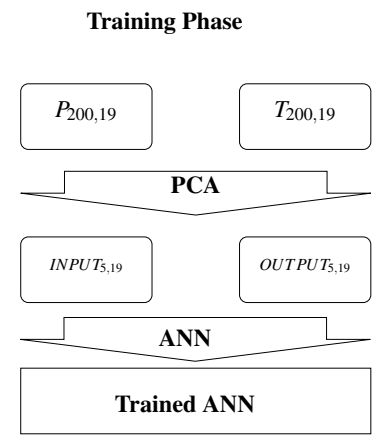

(a)
Testing Phase

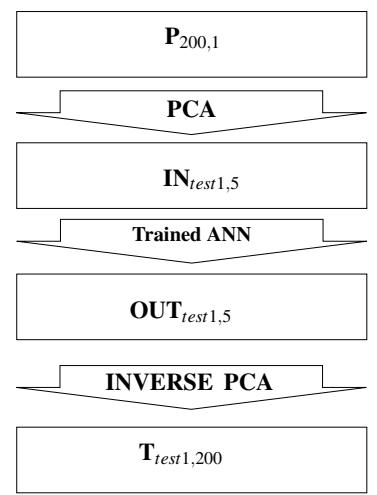

(b)

Fig. 5. (a) The training phase; (b) The testing phase: the estimated Bhattacharyya statistics are fed to the network and the corresponding LV cavity areas are predicted

\subsection{Estimating the Cardiac Ejection Fraction from Image Statistics}

Let $V_{s}$ and $V_{d}$ denote the smallest (end-systolic) and largest (end-diastolic) volumes of the LV in a cardiac cycle, respectively ( Fig. 4(b)). The cardiac ejection fraction, $E F$, is given by: $E F=\frac{V_{d}-V_{s}}{V_{d}}$. The numerator measures the blood volume pumped by the left ventricle. We computed $V_{s}$ and $V_{d}$ by integrating the computed LV cavity areas in the sagittal direction.

\section{Experimental Evaluations and Comparisons}

A set of 2D short-axis cine magnetic resonance (MR) images of 20 subjects were acquired through the cardiac cycle on a $1.5 \mathrm{~T}$ scanner with fast-imaging employing steadystate acquisition (FIESTA) image sequence mode. The acquisition parameters were as follows: $\mathrm{TR}=2.98 \mathrm{~ms}, \mathrm{TE}=1.2 \mathrm{~ms}$, flip angle=30 degree, and slice thickness $=10 \mathrm{~mm}$. Each subject's dataset consists of 20 frames throughout the cardiac cycle, each comprising 10 slices.

We used the proposed method to automatically compute the LV cavity areas, thereby estimating the LV cavity volumes and ejection fractions in each of the 20 subjects. We proceeded to a leave-one subject-out validation approach, where the training used to compute the volumes of each subject is based on the other 19 subjects. The obtained volumes and ejection fractions were evaluated quantitatively by comparing them with those obtained from independent manual segmentation by an expert. Fig. 6 (a) depicts the computed LV cavity volumes for all 20 patients versus those obtained from the independent manual segmentations, as well as the identity line, which indicates an excellent correlation between manually and automatically computed volumes. In the next

\footnotetext{
${ }^{4}$ The dataset contains normal and abnormal cases.
} 


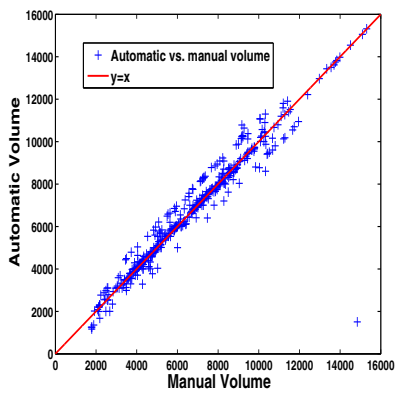

(a)

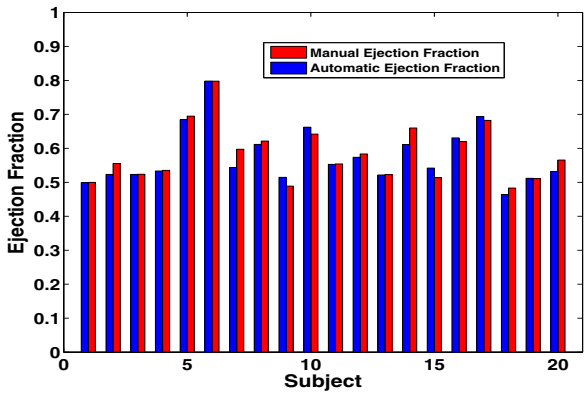

(b)

Fig. 6. (a) Automatic versus manual cavity volumes; (b) Automatic and manual EFs in 20 subjects

step, the estimated cavity volumes were used to estimate the $E F$ s for all 20 subjects. Let $E F_{A}$ be a vector containing the 20 automatically estimated $E F$ s and $E F_{M}$ a vector of the same size containing the $E F$ s obtained form manual segmentations. Fig. 6 (b) depicts $E F_{A}$ and $E F_{M}$, and confirms that the $E F$ s computed with the proposed method are very close to those obtained from independent manual segmentations. We evaluated the conformity between the manually and automatically computed $E F$ s (Table 1). First we evaluated the correlation coefficient, $\operatorname{CorrCoeff}$, which measures the correlation between $E F_{A}$ and $E F_{M}$. The range of CorrCoeff is [0,1], where 1 indicates a perfect fit between the vectors. The proposed method yielded a CorrCoeff of 0.9635, which indicates a high conformity between manual and automatic ejection fractions. We then evaluated the mean and standard deviation of the norm of the difference between $E F_{A}$ and $E F_{M}$ : Diff $f_{E F}=\left\|E F_{A}-E F_{M}\right\|$. The very low mean and standard deviation (std) of $\operatorname{Diff} f_{E F}$ (Table 1) indicates a high conformity between manual and automatic ejection fractions. We used a parametric test (two-tailed t-test) to estimate the conformity

Table 1. Statistical measures of the conformity between automatically and manually computed EFs and computation time (in seconds)

\begin{tabular}{|c|c|c|c|c|}
\hline CorrCoeff $\left(E F_{A}, E F_{M}\right)$ & mean $\left(\operatorname{Diff} f_{E F}\right)$ & $\operatorname{std}\left(\operatorname{Diff} f_{E F}\right)$ & $C P U(s)$ & $P-$ value $(t-t e s t)$ \\
\hline 0.9635 & 0.0160 & 0.0163 & 0.2087 & 0.1778 \\
\hline
\end{tabular}

between manually and automatically estimated ejection fractions that indicated the differences between $E F_{A}$ and $E F_{M}$ were not statistically significant $(P=0.178)$.

Figs 7 depicts automatically and manually computed volumes for three subjects. Fig. 7(a) shows the best estimation in the 20 subjects, which corresponds to the lowest error, i.e., the lowest absolute difference between manually and automatically computed volumes. Fig. 7 (b) corresponds to the medium error (the medium estimation in the 20 subjects), and Fig. 7 (c) to the highest error (the worst estimation in the 20 subjects). The computation time is reported in Table 1 . On a $2.2 \mathrm{GHz}$ machine, a non-optimized MATLAB implementation took 0.2087 seconds to estimate the EF per subject. 


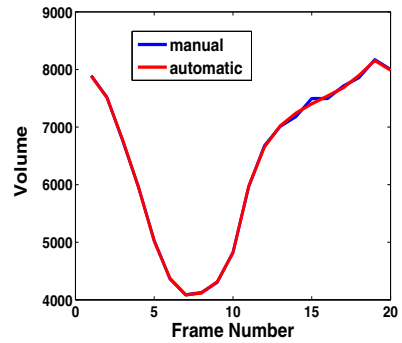

(a)

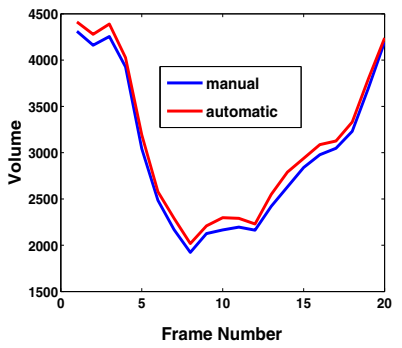

(b)

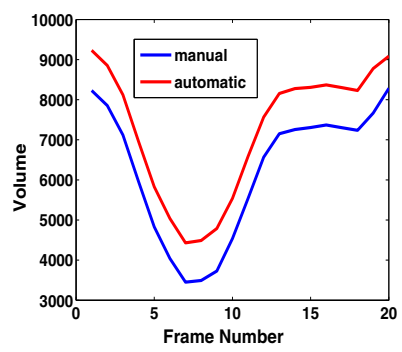

(c)

Fig. 7. Automatic versus manual cavity volumes: (a) the best case in the 20 subjects; (b) the medium case in the 20 subjects; (c) the worst case in the 20 subjects

\section{Conclusion}

This study investigated a real-time method for computing the cardiac EF directly (without segmentation) from image statistics via machine learning. These image statistics were based on the Bhattacharyya coefficients of similarity between image distributions, which were shown to be non-linearly related to the LV cavity areas. An ANN was used to find the relation between the image statistics and the corresponding LV cavity areas in each subject dataset. A comprehensive experimental evaluation over 20 subjects demonstrated an excellent conformity of the automatically estimated EFs to those computed from manual segmentations.

\section{References}

1. Andreopoulos, A., Tsotsos, J.K.: Efficient and generalizable statistical models of shape and appearance for analysis of cardiac MRI. Medical Image Analysis 12(3), 335-357 (2008)

2. Ben Ayed, I., Li, S., Ross, I.: Embedding overlap priors in variational left ventricle tracking. IEEE Transaction on Medical Imaging 28(12), 1902-1913 (2009)

3. Ben Ayed, I., Li, S., Ross, I.G.: A statistical overlap prior for variational image segmentation. International Journal of Computer Vision 85(1), 115-132 (2009)

4. Ben Ayed, I., Punithakumar, K., Li, S., Islam, A., Chong, J.: Left Ventricle Segmentation via Graph Cut Distribution Matching. In: Yang, G.-Z., Hawkes, D., Rueckert, D., Noble, A., Taylor, C. (eds.) MICCAI 2009, Part II. LNCS, vol. 5762, pp. 901-909. Springer, Heidelberg (2009)

5. Fausett, L. (ed.): Fundamentals of neural networks: architectures, algorithms, and applications. Prentice-Hall, Inc. (1994)

6. Fradkin, M., Ciofolo, C., Mory, B., Hautvast, G., Breeuwer, M.: Comprehensive Segmentation of Cine Cardiac MR Images. In: Metaxas, D., Axel, L., Fichtinger, G., Székely, G. (eds.) MICCAI 2008, Part I. LNCS, vol. 5241, pp. 178-185. Springer, Heidelberg (2008)

7. Han, H.C., Martin, R.P., Lerakis, G., Lerakis, S.: Prediction of the left ventricular ejection fraction improvement using echocardiography and mechanical modeling. Journal of the American Society of Echocardiography 18(7), 718-721 (2005) 
8. Lee, H.Y., Codella, N., Cham, M., Weinsaft, J., Wang, Y.: Automatic left ventricle segmentation using iterative thresholding and an active contour model with adaptation on short-axis cardiac MRI. IEEE Transactions on Biomedical Engineering 57(4), 905-913 (2010)

9. Lynch, M., Ghita, O., Whelan, P.: Automatic segmentation of the left ventricle cavity and myocardium in MRI data. Computers in Biology and Medicine 36(4), 389-407 (2006)

10. Michailovich, O., Yogesh, R., Tannenbaum, A.: Image segmentation using active contours driven by the bhattacharyya gradient flow. IEEE Transaction on Image Processing 16(11), 2787-2801 (2007)

11. Pednekar, A., Muthupillai, R., Lenge, V.V., Kakadiaris, I.A., Flamm, S.D.: Automatic identification of the left ventricle in cardiac cine-MR images: Dual-contrast cluster analysis and scout-geometry approaches. Journal of Magnetic Resonance Imaging 23(5), 641-651 (2006)

12. Petitjean, C., Dacher, J.N.: A review of segmentation methods in short axis cardiac MR images. Medical Image Analysis 15(2), 169-184 (2011)

13. Zhu-Jacquot, J., Zabih, R.: Segmentation of the left ventricle in cardiac MR images using graph cuts with parametric shape priors. In: ICASSP, pp. 521-524 (2008) 